\title{
Physical activity in older adults: A cross sectional study
}

\author{
Seyed Abolhassan Naghibi ${ }^{1}$, Fereshteh Rostami ${ }^{2}$, Mahmood Moosazadeh ${ }^{3}$, Seyedeh Somayeh Kazemi $^{4 *}$ \\ 1. School of Health, Mazandaran University of Medical Sciences, Sari, Iran \\ 2. Health Network, Mazandaran University of Medical Sciences, Jouybar, Iran \\ 3. Health Sciences Research Center, Addiction Institute, Mazandaran University of Medical Sciences, Sari, Iran \\ 4. Health Network, Mazandaran University of Medical Sciences, Chaloos, Iran
}

Received: 6 January 2021

Accepted for publication: 1 March 2021

[EPub a head of print-9 March 2021]

Payesh: 2021; 20 (2): 179-190

\begin{abstract}
Objective (s): Aging is one of the most important anthropological phenomena of the 21 st century. Thus due to various benefits of physical activity for health and fitness, it considers being one of the most important factors in healthy aging. This study aimed to investigate physical activity and its determinants in older adults.

Methods: This cross-sectional study was performed on 393 older adults in Mazandaran, Iran, using a multi-stage sampling method. Inclusion criteria included elderly men and women over 60 years of age and their tendency to participate in the study. The data were collected using a demographic questionnaire, the physical activity questionnaire for older adults (CHAMPS), incentive factors, and barriers to physical activity. Descriptive statistics, t-test, and regression were used to analyze the data.

Results: The age of participants ranged from 60-94 years. The results showed that $25.96 \%$ of the older adults were sedentary and $34.86 \%$ had low-intensity, $38.42 \%$ had a moderate intensity and $0.76 \%$ had high-intensity physical activity. The main factors for physical activity were: The right companion, feeling relaxed, friends' encouragement, meeting friends, the doctor prescribing, and maintaining fitness. Barriers included illness and medical factors, long distances, lack of sports venues, fear of falling and injury, lack of access to equipment and facilities. The results also indicated a relationship between physical activity and variables such as age, living conditions, elderly male occupation, cardiovascular disease, and osteoporosis.

Conclusion: Overall, physical activity in older adults seems to be multifactorial in nature, and a wide range of sociodemographic factors are involved in determining the level of individual activity. The level of physical activity in the present study was low and most participants had low-intensity physical activity. Based on the findings, identifying effective factors and barriers has an important role in promoting physical activity in older adults.
\end{abstract}

Key Words: Physical activity, Enabling Factors, Reinforcing Factors, Inhibitory factors, Older adults

\footnotetext{
* Corresponding author: Health Network, Mazandaran University of Medical Sciences, Chaloos, Iran

E-mail: somayeh.kazemi7@gmail.com
} 


\section{فعاليت جسمانى در سالمندان: يك مطالعه مقطعى}

سيد ابوالحسن نقيبى '، فرشته رستمى ‘ محمود موسى زاده ‘ سيده سميه كاظمى

I. إنشكده بهداشت، دانشگاه علوم يزشكى مازندران، سارى، ايران

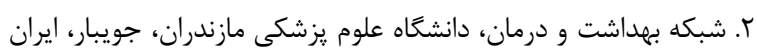

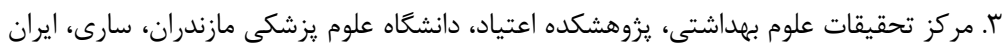

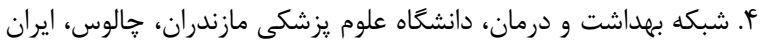

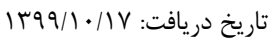

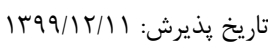

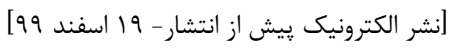

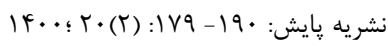

جكيده

مقدمه: هدف از اين مطالعه بررسى فعاليت جسمانى و عوامل تقويت كننده، قادرساز و بازدارنده انجام فعاليت جسمانى در سالمندان بود.

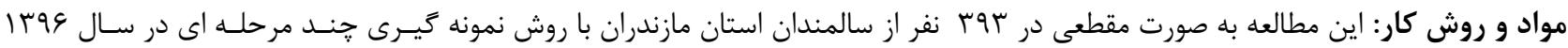

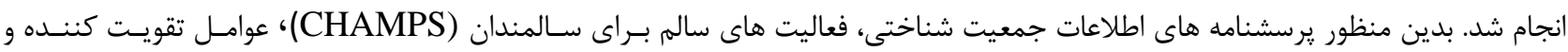

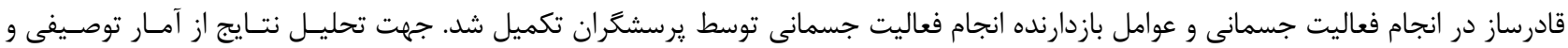

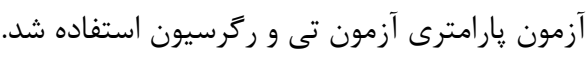

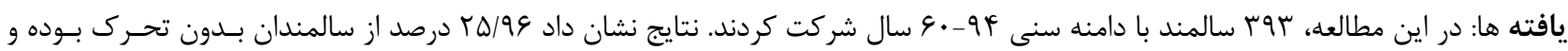

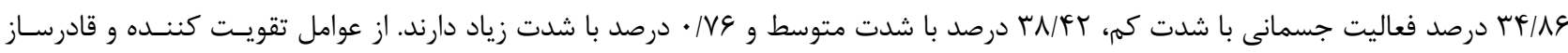

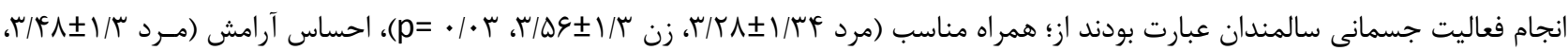

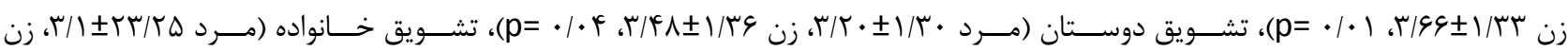
ه/

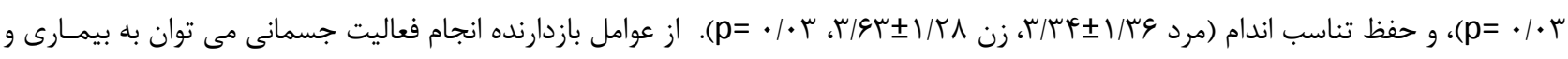

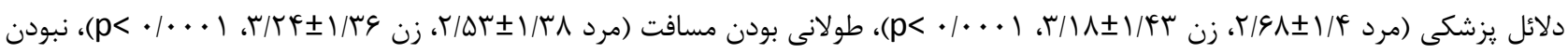

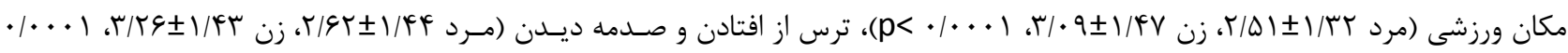

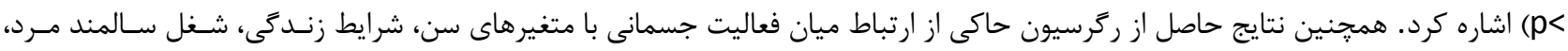

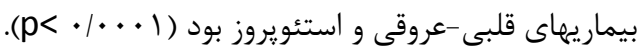

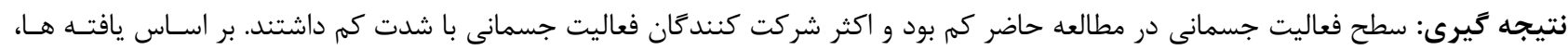

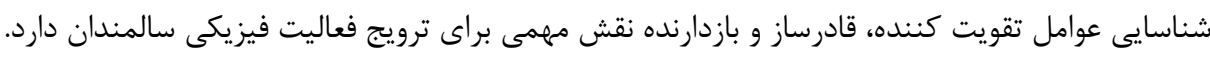

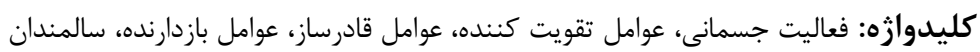


از مطالعات مطرح شـده اسـت [آr، •r]. مطالعات داخلى نيز نشـان

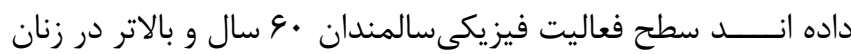

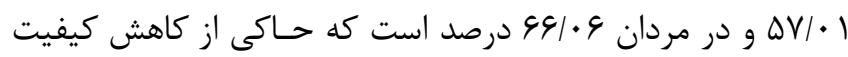

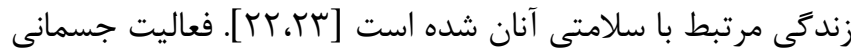

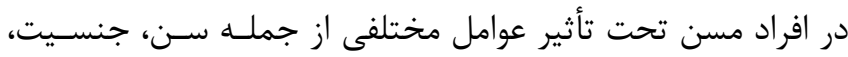

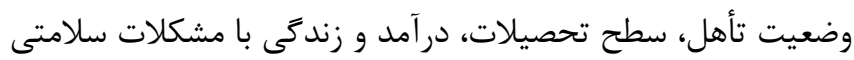

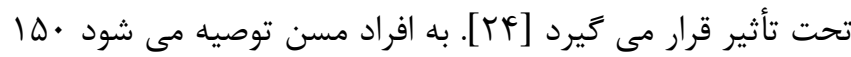

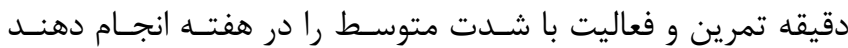

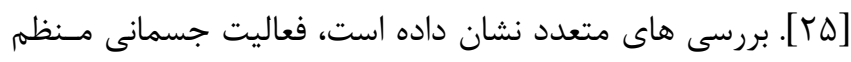

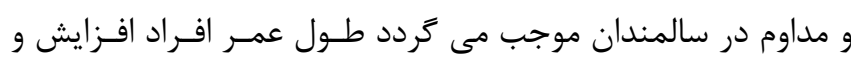

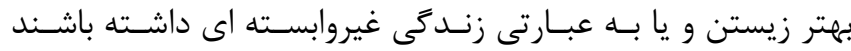

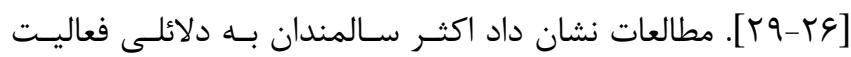

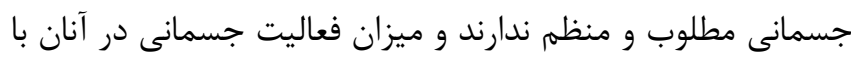

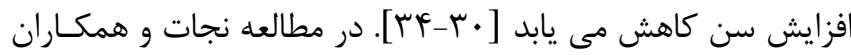

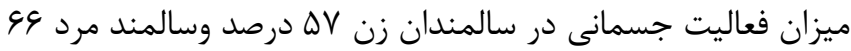

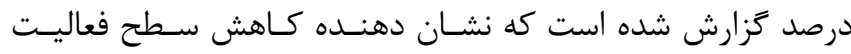

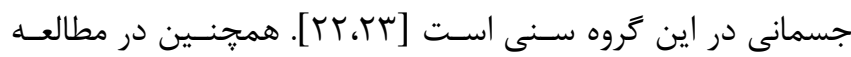

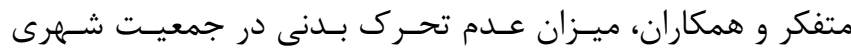

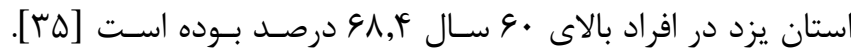

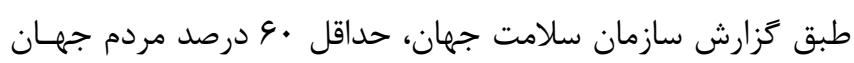
مقدار توصيه شده فعاليت جسمانى را كه جهت فوايد سلامت مـاند

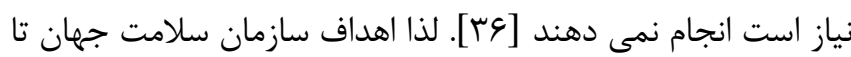

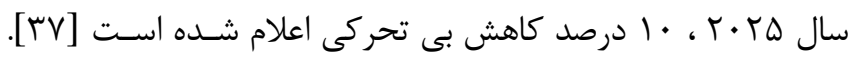

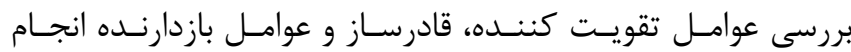

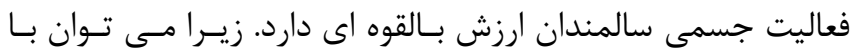

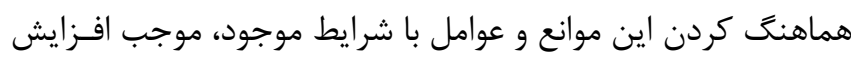

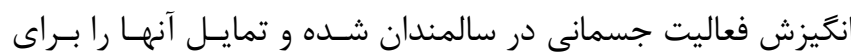

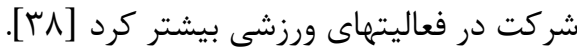

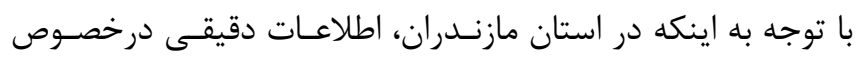

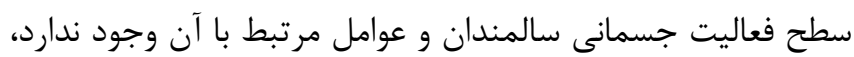
مطالعه حاضر به بررسى سطح فعاليت جسمانى سالمندان و ارزيـابى

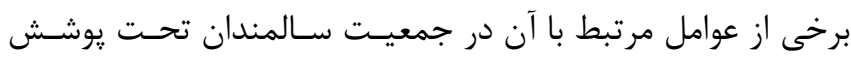

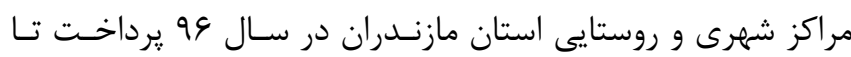

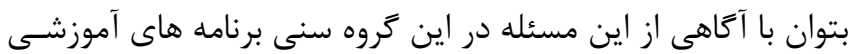
و ورزشى جهت ارتقاى سطح فعاليت جسمانى طراحى و تدوين كرد.

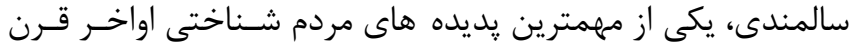

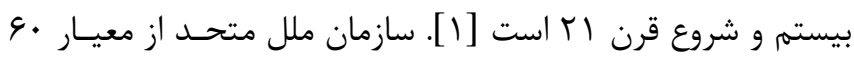

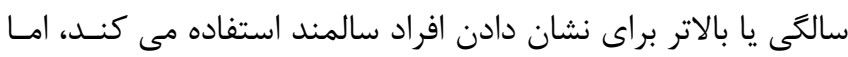

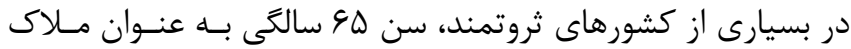

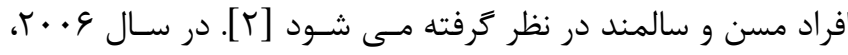

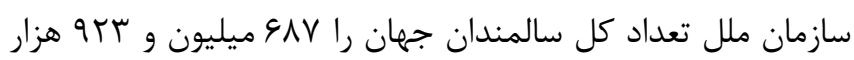

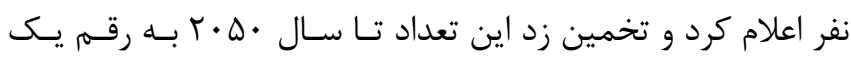

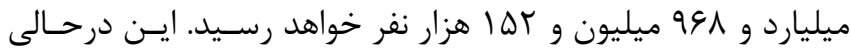

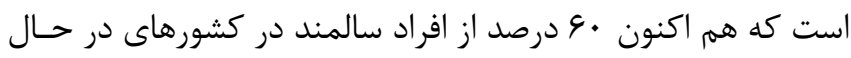
توسعه زندگى مى كنند [ب].

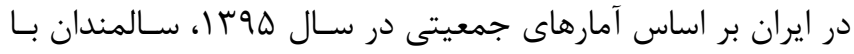

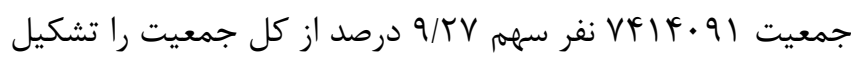

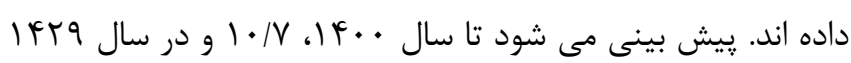

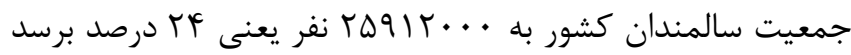

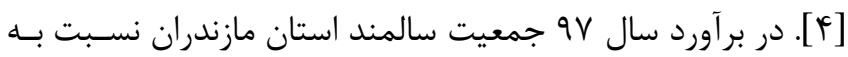

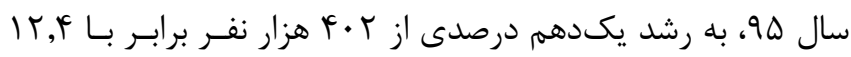

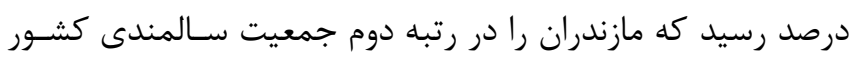

قرار مىدهد [ه].

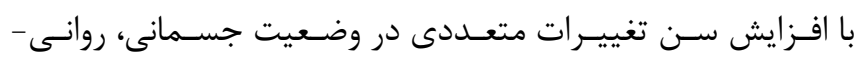

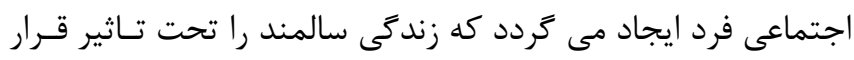

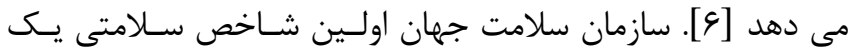

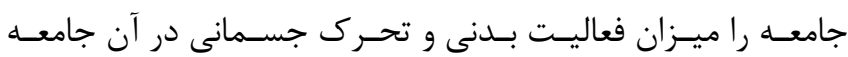

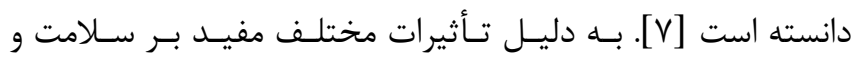

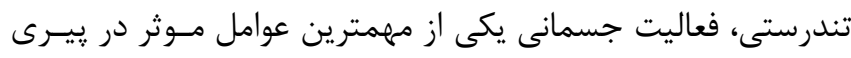

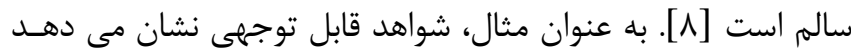

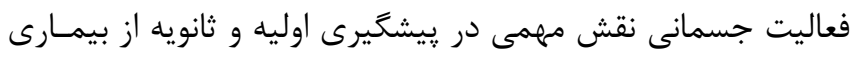

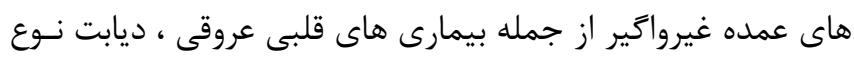

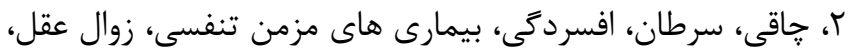

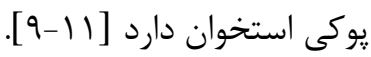

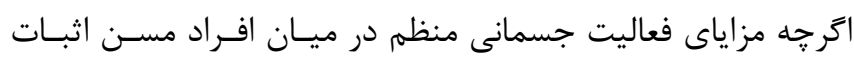

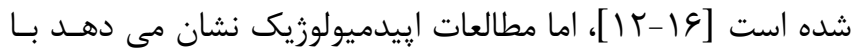

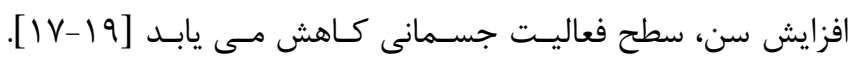

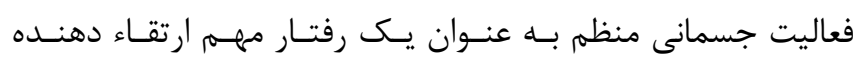

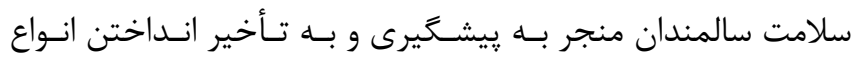

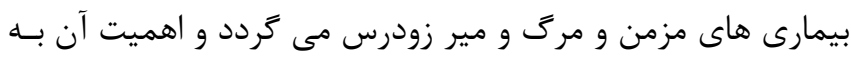
عنوان ابزارى براى تداوم استقلال در افراد سالمند از طريق مردي بسـيارى 
شمس و همكاران (rاس |) در ايران بومى سازى و به تاييد رسـيده

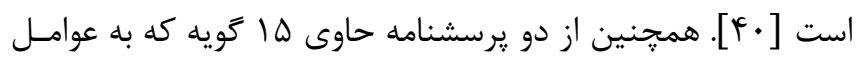

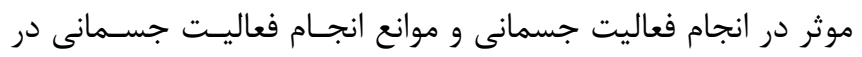

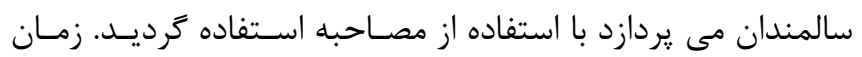

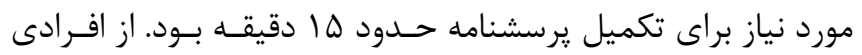
براى تكميل يرسشنامه استفاده شد كه توانايى در برقرارى ارتباط با بال

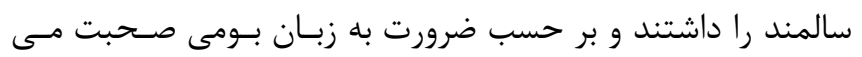

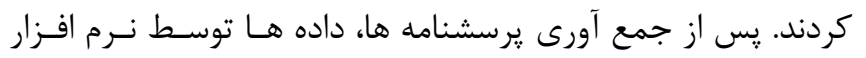

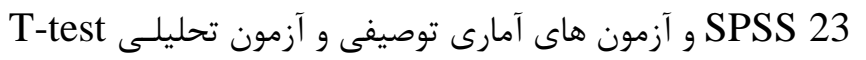

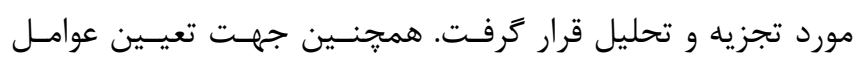
مرتبط با فعاليت جسمانى از آزمون ركرسيون استفاده شد.

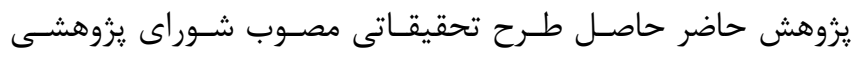

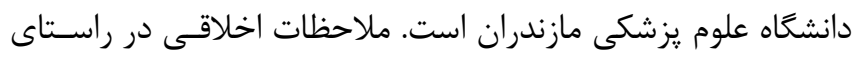

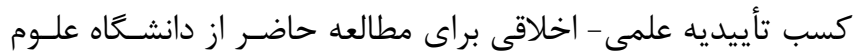

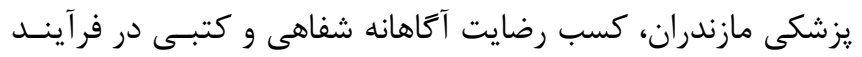

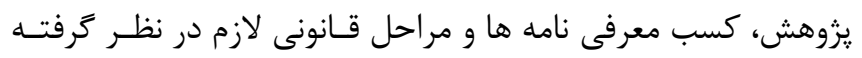

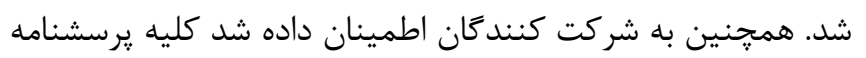

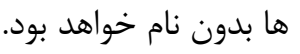

\section{يافتهها}

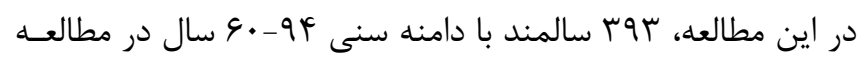

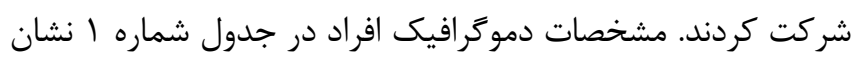

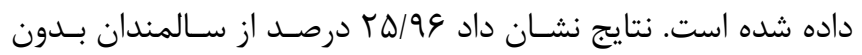
تحرك بوده و د/NG

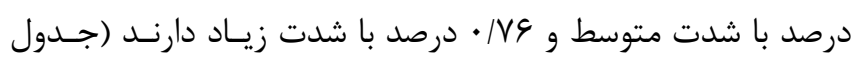

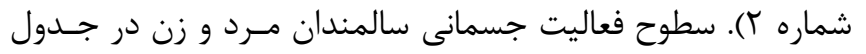

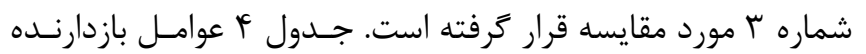

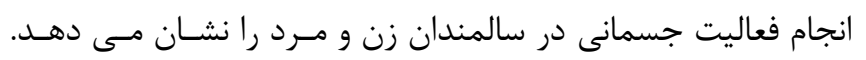

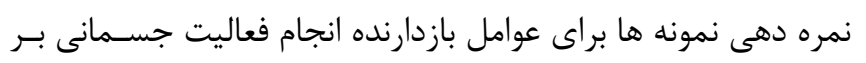

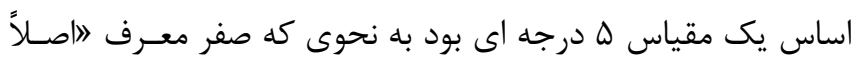

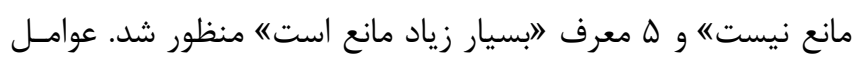

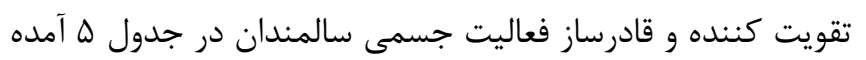

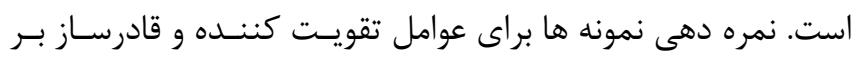

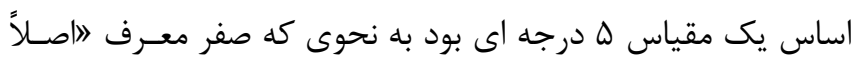

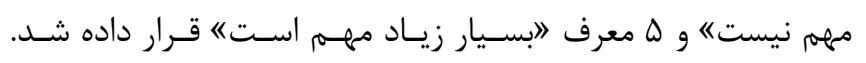

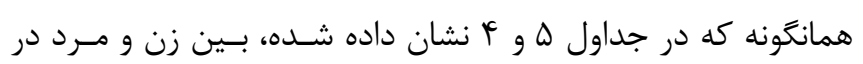

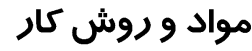

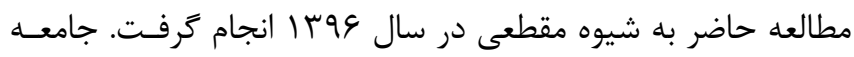

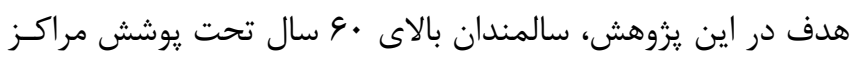

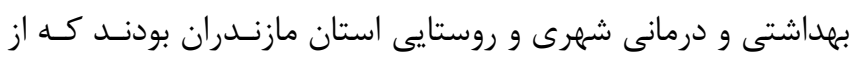

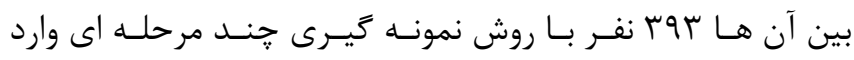

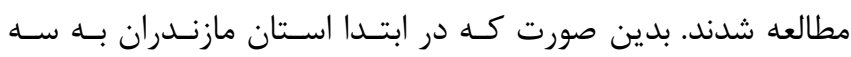

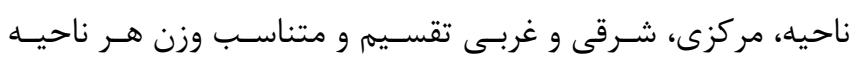

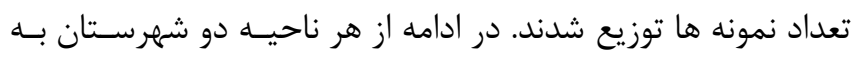

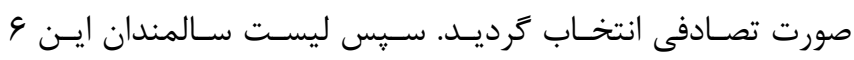

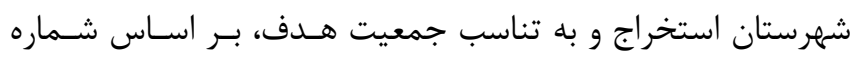

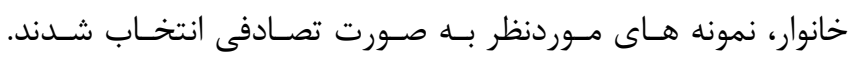

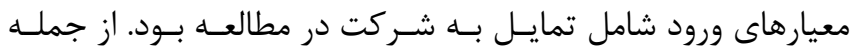

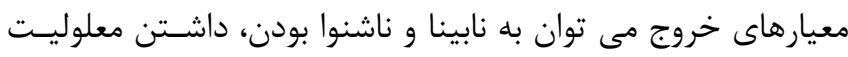

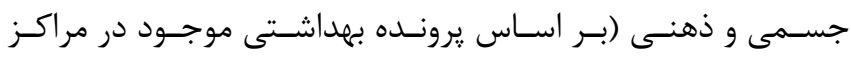

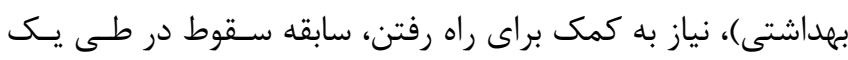

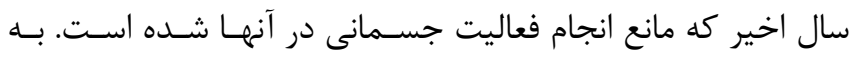

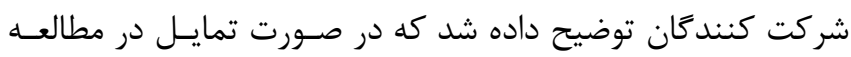

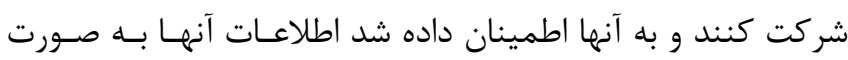

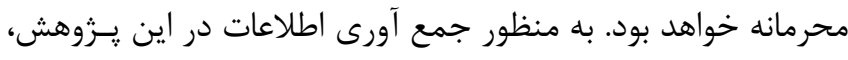

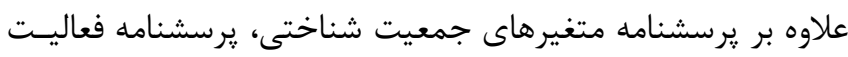

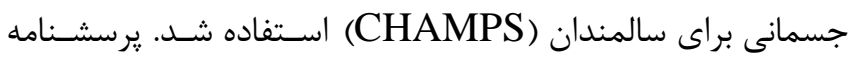

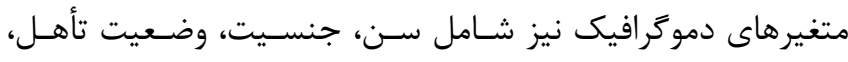

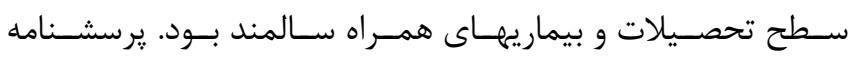
CHAMPS

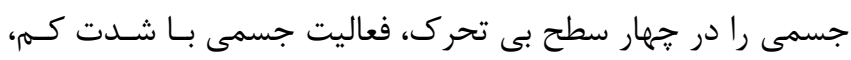

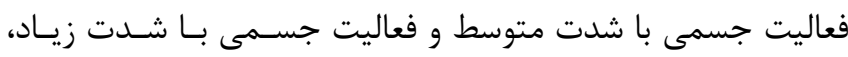

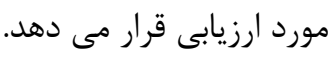

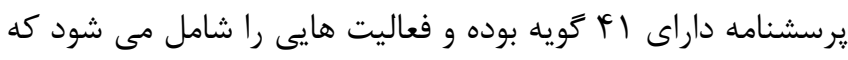

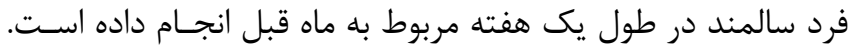

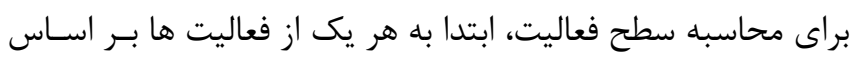

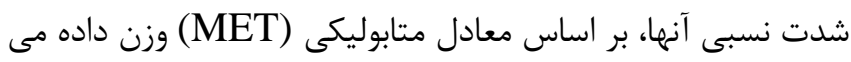

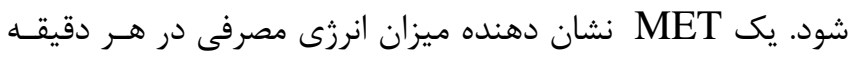

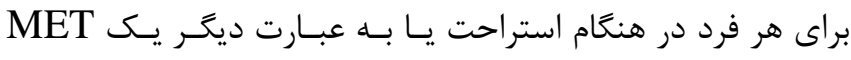

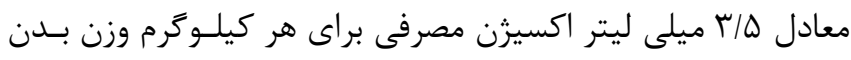
است. بر اين اساس ملاكى نمره كلى، ميزان انرزى مصرفى سالمندان

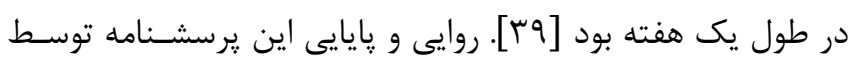


( ( • • • است (جدول 9). به طورى كه فعاليت جسمانى با افزايش

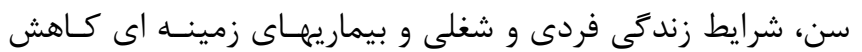

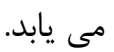

مشوق هاى سلامت و موانع شغلى تفاوت هايى وجود دارد. همجنين

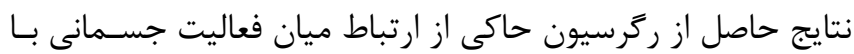

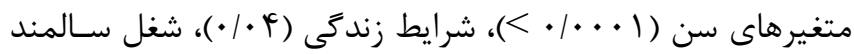

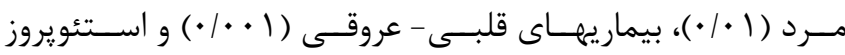

جدول ا: مشخصات دموكرافيك شركت كنندكان

\begin{tabular}{|c|c|c|}
\hline درصد & تعداد & \\
\hline & & جنسيت \\
\hline FV/G & $1 \wedge 9$ & مرد \\
\hline \multirow[t]{2}{*}{$\Delta T / F$} & $r \cdot \varphi$ & زن \\
\hline & & محل سكونت \\
\hline FT/T & 199 & شهر \\
\hline \multirow[t]{2}{*}{$\Delta \mathrm{V} / \Lambda$} & trt & روستا \\
\hline & & سطح تحصيلات \\
\hline$\Lambda N / \Lambda$ & req & زير دييلم \\
\hline \multirow[t]{2}{*}{$11 / r$} & rq & دييلهم و بالاتر \\
\hline & & وضعيت تاهل \\
\hline $1 / \Delta$ & 4 & مجرد \\
\hline$V T / T$ & rAK & متاهل \\
\hline TF/V & $\Delta V$ & بيوه \\
\hline \multirow[t]{2}{*}{$1 / 0$} & 9 & مطلقه \\
\hline & & شرايط زندكى \\
\hline IV/T & 91 & تنها \\
\hline \multirow[t]{2}{*}{$\Lambda T / V$} & rTa & با همراه \\
\hline & & شغل سالمند زن \\
\hline $9 \Delta / q^{\circ}$ & $f \cdot \Delta$ & خانه دار \\
\hline$r / 1$ & ir & بازنشسته \\
\hline$\cdot / r$ & 1 & كشاورز \\
\hline \multirow[t]{2}{*}{$1 / \pi$} & $\Delta$ & كاركر \\
\hline & & شغل سالمند مرد \\
\hline$\Delta 9$ & THT & بيكار \\
\hline 1911 & 99 & بازنشسته \\
\hline $1 N / 9$ & vr & كشاورز \\
\hline$r / \Lambda$ & 11 & كاركر \\
\hline$\cdot / \Lambda$ & r & كارمند \\
\hline \multirow[t]{2}{*}{ r } & $\wedge$ & آزاد \\
\hline & & مصرف دخانيات \\
\hline lf & $\Delta \Delta$ & دارد \\
\hline \multirow[t]{2}{*}{$\wedge 9$} & ג & 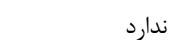 \\
\hline & & سيكار \\
\hline $1 \cdot / 4$ & \&i & بله \\
\hline 19/9 & rar & خير \\
\hline & & قليان \\
\hline r & $\wedge$ & بله \\
\hline \multirow[t]{2}{*}{91} & rAD & خير \\
\hline & & ترياك \\
\hline$r / 9$ & If & بله \\
\hline १९/४ & rVq & خير \\
\hline$\cdot / 1$ & 1 & له له \\
\hline १९/v & rar & خير \\
\hline
\end{tabular}


$r F / T$

$\mathrm{VD} / \mathrm{A}$

$4 q / 4$

$\Delta \cdot 19$

$r T / 9$

$\mathrm{V} / \mathrm{I}$

TI/S

VN/F

ए/

$99 / 9$

r/A

q $/ T$

$0 / 1$

$q 4 / 9$

19/1

$\Lambda r / r$

$r / 9$

$99 / 4$

$1 \cdot / 4$

$19 / 9$

./VG

rV/VT

FI/V

19/AF

$\Delta / 9$

P/T
90

ras

194

199

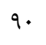

r.r

$\Lambda \Delta$

$r \cdot \Lambda$

it

rNI

11

rAT

$r$.

rvr

94

rTV

if

rvq

i)

rar

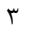

1.9

ist

VA

rT
ديابت

دارد

ندارد

فشار خون

دارد

ندارد

قلبى عروقى ندارد

دارد

ندارد

جربى خون

دارد

ندارد

آرتريت

دارد

ندارد

استئويروز

دارد

ندارد

بيمارى مزمن ريوى ندارد

دارد

ندارد

اختلالات اسكلتى عضلانى

دارد

ندارد

بيرون زدكى ديسك

دارد

ندارد

سابقه سقوط

دارد

ندارد

BMI

كم وزن

طبيعى

اضافه وزن إني

جاقى درجه

جاقى درجه

جاقى درجه

جدول ז: فعاليت جسمانى در سالمندان

\begin{tabular}{|c|c|c|}
\hline درصد & تعداد & \\
\hline Гब/৭६ & $1 \cdot r$ & بى تحرى \\
\hline rN/Ft & 101 & فعاليت جسمانى با شدت كم \\
\hline rY/AS & irv & فعاليت جسمانى با شدت متوسط \\
\hline •/Ve & r & فعاليت جسمانى با شدت زياد \\
\hline
\end{tabular}


سال بيستم، شماره دوم، فروردين - ارديبهشت .. سF

$\int_{\infty}^{\infty}$

سيده سميه كاظمى و همكاران

فعاليت جسمانى در سالمندان ...

جدول ؟ّ: مقايسه فعاليت جسمانى در سالمندان مرد و زن

\begin{tabular}{|c|c|c|c|c|}
\hline \multicolumn{2}{|c|}{ سالمند زن } & \multicolumn{3}{|c|}{ سالمند مرد } \\
\hline درصد & تعداد & درصد & تعداد & \\
\hline$|V / T|$ & 91 & $N / 9 \Delta$ & re & بى تحرك \\
\hline$r r / q$ & q. & $1 Q / \Delta T$ & 41 & فعاليت جسمانى با شدت كم \\
\hline IQ/QT & 91 & $19 / \pi 4$ & ve & فعاليت جسمانى با شدت متوسط \\
\hline . & . & - /ve & r & فعاليت جسمانى با شدت زياد \\
\hline
\end{tabular}

*t-test $(\overline{\mathrm{P}<0.0001)}$

جدول f: عوامل بازدارنده انجام فعاليت جسمانى در سالمندان

\begin{tabular}{|c|c|c|c|c|c|}
\hline \multicolumn{3}{|c|}{ سالمند زن (Y.9) } & \multicolumn{3}{|c|}{ سالمند مرد (IAV) } \\
\hline $\mathrm{P}^{*}$ & انحراف معيار & ميانكَين & انحراف معيار & ميانكين & \\
\hline$<.|\ldots|$ & I/Fr & $r / / \Lambda$ & $1 / 4$ & $r / 9 \Lambda$ & لائل يزشكى \\
\hline$<.|\ldots|$ & $1 / 44$ & $r / T F$ & $1 / \pi \wedge$ & $r / \Delta T$ & طولانى بودن مسافت \\
\hline$<.|\ldots|$ & $1 / 4 \mathrm{~V}$ & $r / \cdot 9$ & $1 / \pi T$ & $T / Q \mid$ & نبودن مكان ورزشى \\
\hline $1 . .4$ & $1 / r F$ & $r / l f$ & $1 / 1 \mathrm{~V}$ & $1 / V Y$ & تخَهدارى از نوه و نداشتن وقت \\
\hline $.1 \cdot 1$ & $1 / r \Delta$ & $1 / v \wedge$ & $1 / 44$ & $r / l$. & شغل ياره وقت \\
\hline .19 & $1 / \pi 4$ & $r / 1 \Delta$ & $1 / \pi 1$ & $T / 19$ & 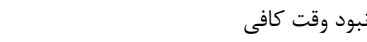 \\
\hline$<.|\ldots|$ & $1 / 4 T$ & $r / r \varphi$ & $1 / 44$ & T/QT & ترس از افتادن و صدمه ديدن \\
\hline.$/ T$ & $1 / 4 \Delta$ & t/AF & $1 / 49$ & r/99 & عدم وجود ورزش مناسب \\
\hline I..Y & 1/ar & r/Aq & $1 / 49$ & t/FT & در دسترس نبودن وسيله اياب و ذهاب \\
\hline$\cdot / r$ & $1 / \pi 4$ & $r / \Delta V$ & $1 / 79$ & t/FE & علاقه نداشتن به فعاليتهاى ورزشى \\
\hline$\cdot 11$ & $1 / 44$ & r/GT & $1 / 79$ & t/AT & حساس تنبلى \\
\hline$\cdot / \cdot v$ & $1 / 44$ & r/qr & $1 / \% \Lambda$ & r/99 & نداشتن همراه مناسب براى ورزش كردن \\
\hline $.1 . .9$ & $1 / 4 \Delta$ & $r / 9 \Lambda$ & $1 / \pi \omega$ & $r|q|$ & خستكى ناشى از انجام فعاليت ورزشى \\
\hline $.1 . \cdot 1$ & $1 / 49$ & $r / l$. & 1/4T & $r / 9$. & ورزش كردن در هواى كرم با لباس يوشيده \\
\hline$\% \Delta$ & $1 / \Delta V$ & $r / 9 \Delta$ & $|/ 4|$ & $r / \Delta r$ & عدم در دسترس بودن وسايل \\
\hline
\end{tabular}

*t-test

جدول ه: عوامل تقويت كننده و قادرساز انجام فعاليت جسمانى سالمندان

\begin{tabular}{|c|c|c|c|c|c|}
\hline \multicolumn{3}{|c|}{ سالمند زن (Y+9) } & \multicolumn{3}{|c|}{ سالمند مرد (IAV) } \\
\hline $\mathrm{P}^{*}$ & انحراف معيار & ميانكَين & انحراف معيار & ميانكَين & \\
\hline$\cdot / 4$ & $1 / \pi V$ & T/r & $1 / 4 \mathrm{~V}$ & $r / / V$ & حفظ استقلال و عدم وابستى \\
\hline$\cdot 11$ & $1 / \pi 9$ & r/4 & $1 / 4$ & $r / 19$ & مكانات ورزشى مناسب \\
\hline$\cdot / 4$ & $1 / \pi 9$ & $r / \uparrow_{1}$ & $1 / 4 T$ & 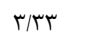 & در دسترس بودن مراكز ورزشى و ياركها \\
\hline$\cdot 19$ & $1 / \pi \Delta$ & $r / r \cdot$ & $1 / \pi v$ & $r / T s$ & بيكار بودن \\
\hline$\cdot 19$ & $1 / T V$ & $r / \uparrow \Delta$ & $1 / 41$ & $r / \pi q$ & سن بالا \\
\hline$\cdot / 4$ & $1 / T V$ & $r / r 4$ & $1 / \Gamma \Lambda$ & $r / 19$ & جلوكيرى از خستخى \\
\hline . & $1 / \Gamma \Lambda$ & r/va & $1 / r \Lambda$ & $r / 4 \Delta$ & تجويز يزشك \\
\hline.$/ \cdot r$ & $1 / \Gamma \Lambda$ & r/q & $1 / \pi 4$ & $r / r 4$ & حفظ تناسب اندام \\
\hline$\cdot / 1$ & $1 / T V$ & $r / V^{4}$ & $1 / \pi 4$ & r/ar & كاهش وزن \\
\hline. & $1 / \pi$ & $r / \Delta 9$ & $1 / \pi 4$ & $r / T \Lambda$ & عمراه مناسب \\
\hline.$/ \cdot 1$ & $1 / \pi r$ & $r / 98$ & $1 / \pi 1$ & $r / 4 \Lambda$ & حساس آرامش \\
\hline$\cdot 1 \cdot 4$ & $1 / \pi 4$ & $r / 4 \wedge$ & $1 / \pi$ & $r / r$. & ت تشويق دوستان \\
\hline $.1 \cdot 1$ & $1 / \pi \Delta$ & $r / \Delta \varphi$ & $1 / \pi \Delta$ & r/Tr & تشويق خانواده \\
\hline.$/ \cdot r$ & $1 / \pi r$ & $r / \Delta \cdot$ & $1 / \pi 1$ & $r / r)$ & ملاقات با دوستان \\
\hline$\cdot / r$ & $1 / \pi r$ & $r / r$. & $1 / \pi r$ & $r / .9$ & خريد مايحتاج زندگى \\
\hline
\end{tabular}




\begin{tabular}{|c|c|c|c|}
\hline P* & SE & 及 & \\
\hline$<\cdot|\cdots|$ &.$/ 48$ & $-T / V T$ & سن \\
\hline$\cdot 1 \cdot r$ & $11 / \cdot F$ & TT/DF & شرايط زندگى \\
\hline.$/ \cdot 1$ & $F / r)$ & $G / / \Delta \varphi$ & شغل سالمند مرد \\
\hline$\cdot 1 \cdot \cdot 1$ & $r / V V$ & - & بيماريهاى قلبى - عروقى \\
\hline$\cdot 1 \cdot .1$ & $\Gamma / 10$ & $-Y T / M$ & استئويروز \\
\hline
\end{tabular}

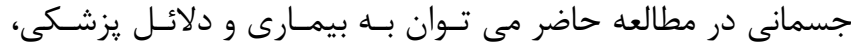

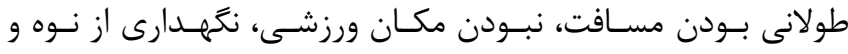

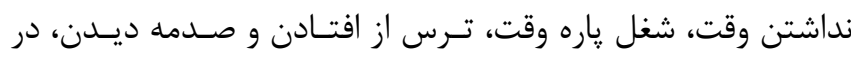
دسترس نبودن وسيله اياب و ذهاب، خستكى ناشى از انجام فعاليـت

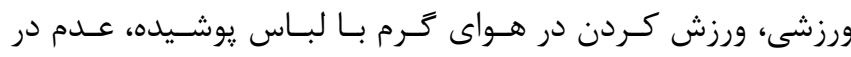

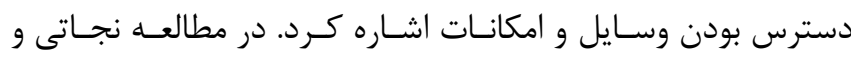

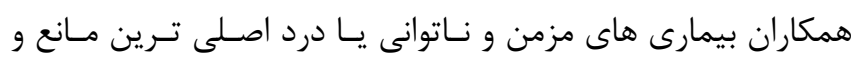

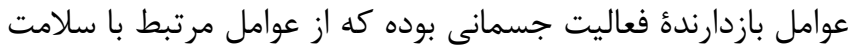

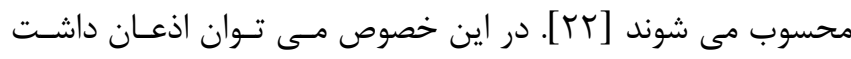

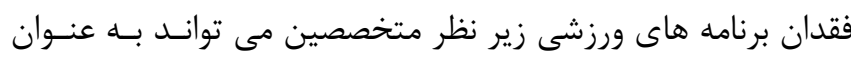
عامل بازدارندة فعاليت فيزيكى مطرح باشد. در مطالعه مرورى انجـام

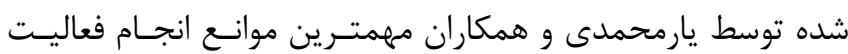

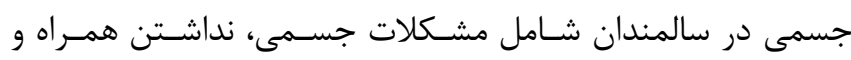

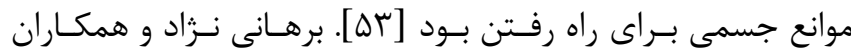

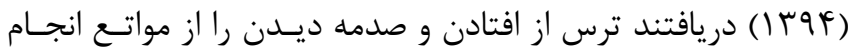

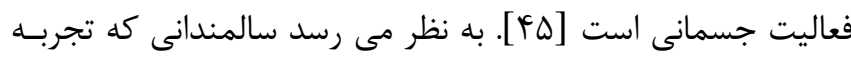
افتادن دارند، ممكن است فعاليت هايشان به خاطر تـرس از افتـادن تحت تاثير قـرار كيــد. ارتقـاى ميـزان فعاليـت جسـمانى در خـروه

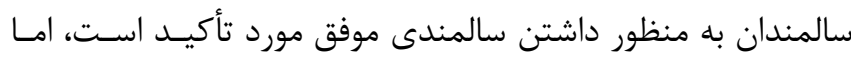

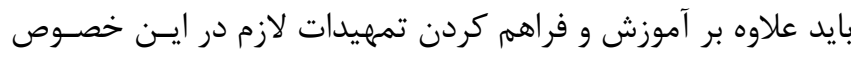

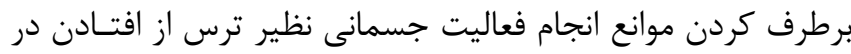

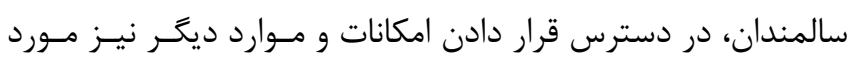

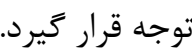
يافته هاى مطالعهُ حاضر نشان داد عوامل تقويـت كنــــده و قادرسـاز فعاليت جسمى سالمندان عبارت بودند از: همراه مناسـب، احسـاس هاس

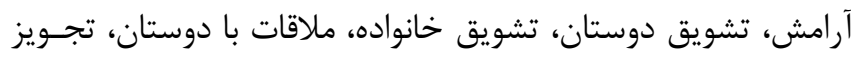

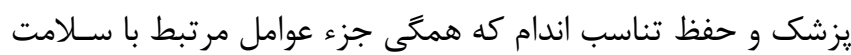

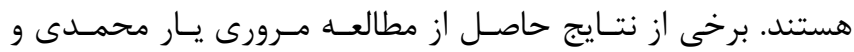

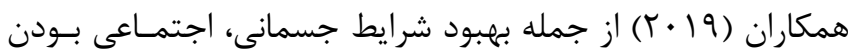
(ملاقات با دوستان) و مناسب بودن محيط فيزيكى فرد جهت انجـام المان

\section{بحث و نتيجه گيرى}

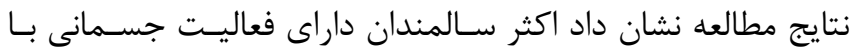

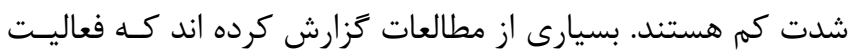

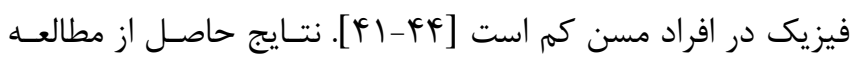

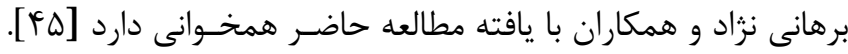

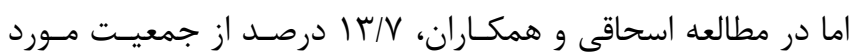

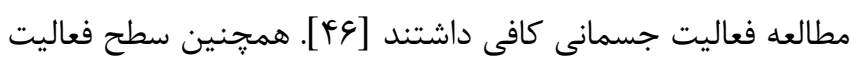

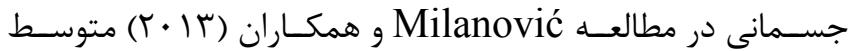

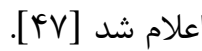

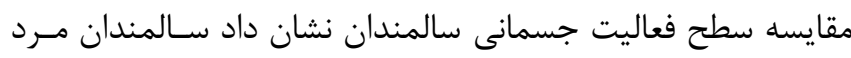

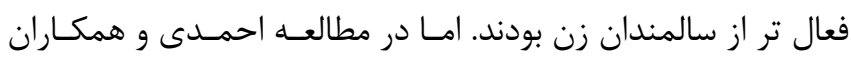

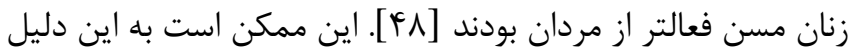

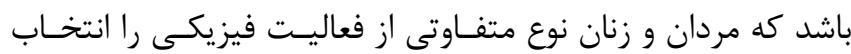

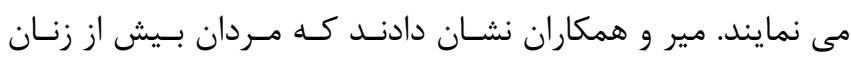

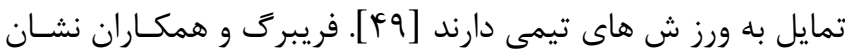

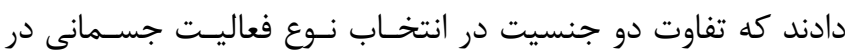

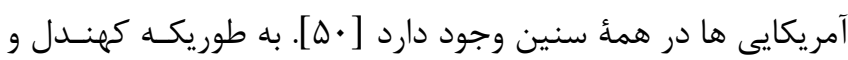

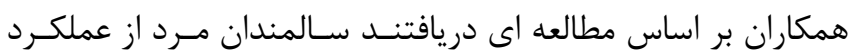

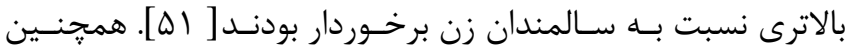

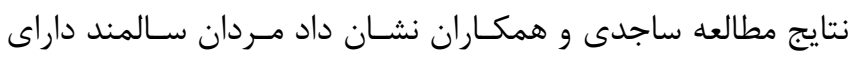

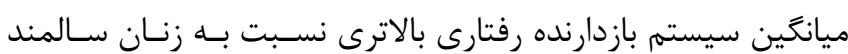

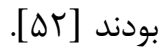
عدم فعاليت در سالمندان از مشكلات شايع اين دوران بـوده و علـل

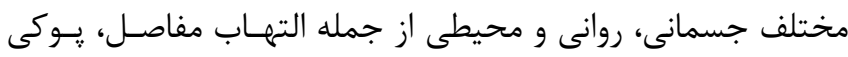

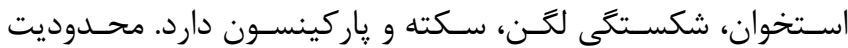
حركتى در سالمندان به دليل نار احتى و دردهاى همراه بــه سـلامت

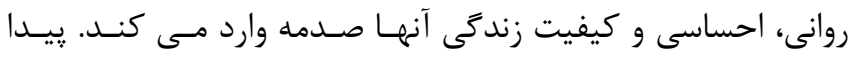

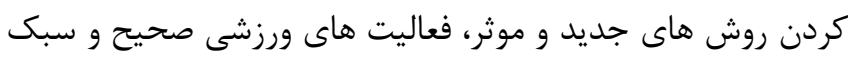

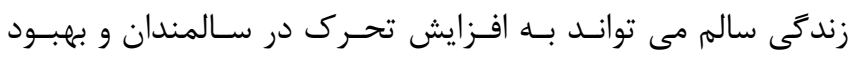

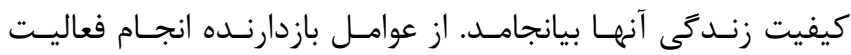


از محدوديت هاى مطالعه عدم بررسى ميدانى عوامـل محيطى مسى

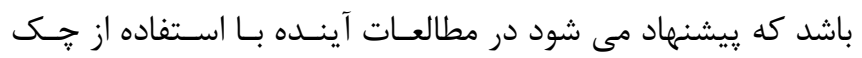
ليست هاى مربوطه اين موضوع مورد بررسى قرار زيرد.

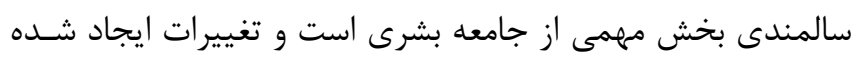

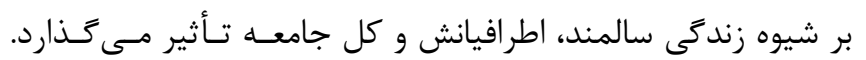

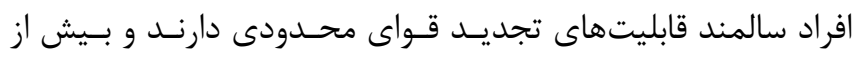

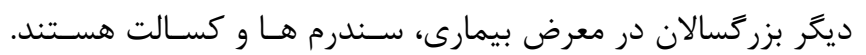

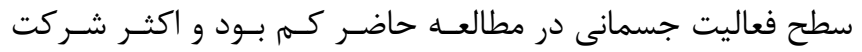
كنندكان فعاليت جسمانى با شدت كم داشتند. بر اساس يافتـهـ هـا،

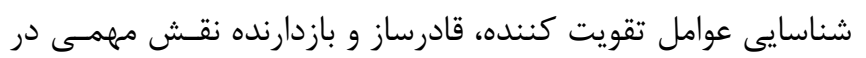
ترويج فعاليت جسمى سالمندان دارد.

\section{سمم نويسند}

ابوالحسن نقيبى: مجرى اول طرح و استاد راهنما

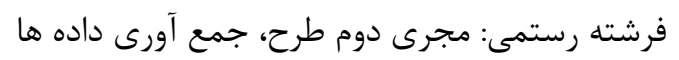
محمود موسى زاده: همكار طرح، مشاور آمارى

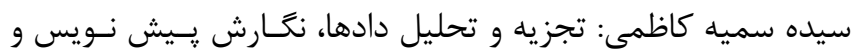
نسخه نهايى مقاله

\section{تشكر و قدردانى}

يزوهش حاضر، طرح مصـوب دانشـعاه علـوم يزشـكى مازنـدران اسـت.

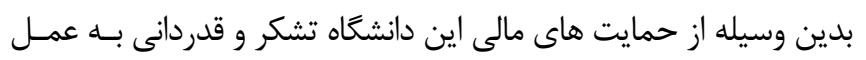

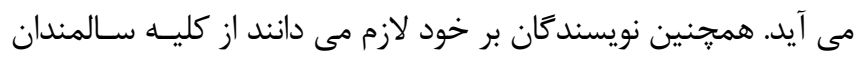

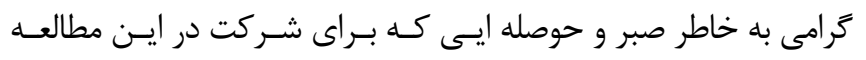
داشته اند، تقدير و تشكر نمايند.

\section{منابع}

1. Alhani F, Asadi Noghabi A, Peyrovi H. The concept of health in the elderly people: A literature review. Iran Journal of Nursing 2012; 25: 62-71 [Persian] 2. Ajh N, Mehrtash B, Javadi AM. Effect of education and social support on quality of life among elderly living in nursing homes 2010. Journal of Qazvin University of Medical Sciences 2012; 16: 46-52

3. Habibi A, Nikpour S, Seyed Shohadaii M, Hagani H. Quality of life and status of physical functioning among elderly in west region of Tehran: A crosssectional survay. Iran Journal of Nursing 2008; 21: 2939 [Persian]
فعاليت جسمى با نتايج مطالعه حاضر همخوانى داشت [مس]. بر اين اساس به نظر مسى رســـ بــراى تـرويج فعاليـت جسـمى ســالمندان بهترين عامل معرفى اثرات فعاليـت جسـمى بـر سـلامت جسـمى و

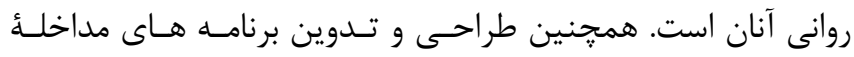
تمرينى مى تواند منجر به افزايش خود كارآمدى و اعتماد به نفس درئ درو

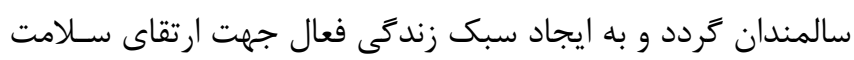
جسمى روانى و كيفيّت زندگى سالمندان كمك نمايد.

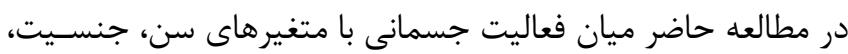

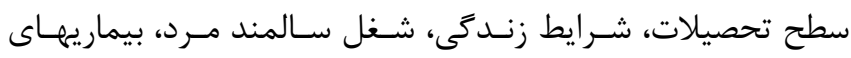

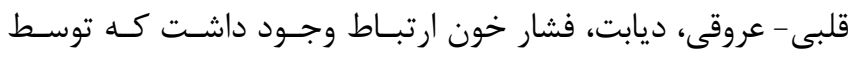

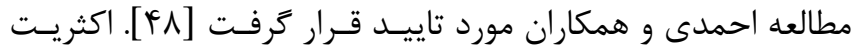

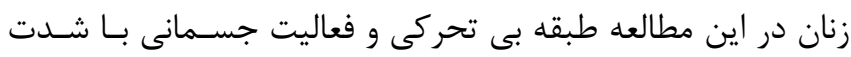

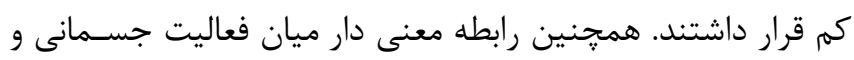

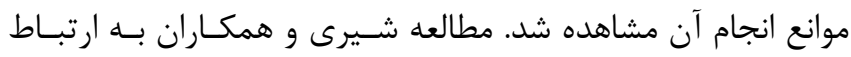

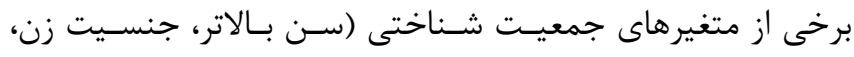
سطح تحصـيلات يـايين، بازنشسـتكى و وضـعيت مجــرد يـا بيـوه)،

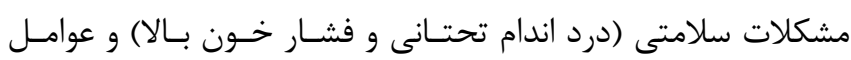

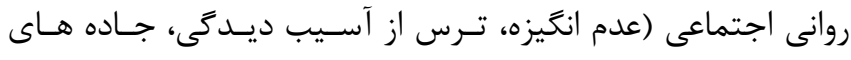

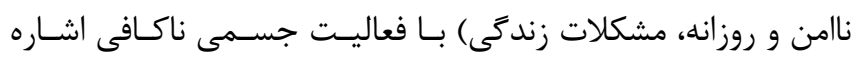
مى كند [FV] به طور كلى، به نظر مى رسد فعاليت جسمانى در افراد مسن ماهيت هند عاملى دارد و طيف وسـيعى از عوامـل اجتمــاعى -جمعيتـى در تعيين سطح فعاليت هاى فردى دخيـل اسـت [FT]

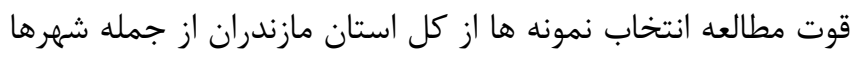

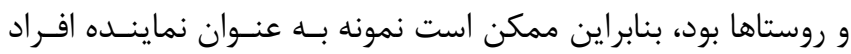
مسن در استان در نظر گرفته شود.

4. Rasel M, Ardalan A. The Future of Ageing and Its Health Care Costs: A Warning for Health System. Yektaweb_Journals 2007;2: 300-5

5. https://www.ilna.news/fa/tiny/news-977936

6. Agamolaei T TS, Hasani L. Self-efficacy, perceived benefits and perceived barriers to regular physical activity Hormozgan University of Medical Sciences. Epidemiology 2007; 4: 9-15

7. Lee C. D, Blair, N, and Jackson, S. Cardiorespiratory fitness, body composition and cardio vascular disease mortality in men. The American Journal of Clinical Nutrition 2000; 69: 373- 80 
8. King AC, King DK. Physical activity for an aging population. Public Health Reviews 2010;32: 401-26

9. Bull FC, Armstrong TP, Dixon T, Ham S, Neiman A, Pratt M. Physical inactivity. In: Ezzati M, Lopez AD, Rodgers A, Murray CJL, Editors. Comparative quantification of health risks: global and regional burden of disease attributable to selected major risk factors. Geneva: World Health Organization 2004. 1: 729-881. apps.who.int

10. Warburton DER, Nicol CW, Bredin SSD. Health benefits of physical activity: the evidence. Canadian Medical Association Journal 2006;174: 801-9

11. Reiner M, Niermann C, Jekauc D, Woll A. Longterm health benefits of physical activity-a systematic review of longitudinal studies. BMC Public Health 2013;13: 1-9

12. Byberg L, Melhus H, Gedeborg R, Sundström J, Ahlbom A, Zethelius B, et al. Total mortality after changes in leisure time physical activity in 50 years old men: 35-year follow-up of population based cohort. BMJ 2009; 338: 1-9. doi: https://doi.org/10.1136/bmj.b688

13. Hamer M, Lavoie KL, Bacon SL. Taking up physical activity in later life and healthy ageing: The English longitudinal study of ageing. British Journal of Sports Medicine 2014;48: 239-43

14. Holme I, Anderssen SA. Increases in physical activity is as important as smoking cessation for reduction in total mortality in elderly men: 12 years of follow-up of the Oslo II study. British Journal of Sports Medicine 2015;49: 743-8

15. Iijima $\mathrm{K}$, Iimuro $S$, Shinozaki $T$, et al; Japanese Elderly Diabetes Intervention Trial Investigator Group. Lower physical activity is a strong predictor of cardiovascular events in elderly patients with type 2 diabetes mellitus beyond traditional risk factors: The Japanese Elderly Diabetes Intervention Trial. Geriatrics \& Gerontology International 2012;12: 7787. DOI: 10.1111/j.1447-0594.2011.00815.x

16. Ueshima K, Ishikawa-Takata $K$, Yorifuji $T$, et al. Physical activity and mortality risk in the Japanese elderly: a cohort study. American Journal of Preventive Medicine 2010;38: 410-418

17. TNS Opinion \& Social. Special Eurobarometer 412: Sport and physical activity. Conducted by TNS Opinion \& Social at the request of the DirectorateGeneral for Education and Culture. Survey coordinated by the Directorate-General for Communication (DG COMM "Strategy, Corporate Communication Actions and Eurobarometer" Unit).
Brussels: TNS Opinion \& Social 2014. DOI: 10.2766/73002.

http://ec.europa.eu/public_opinion/index_en.htm 18. Smith L, Gardner B, Fisher A, Hamer M. Patterns and correlates of physical activity behavior over 10 years in older adults: prospective analyses from the English Longitudinal Study of Ageing. BMJ Open 2015;5, e007423

19. van Stralen MM, De Vries H, Mudde AN, Bolman $C$, Lechner L. Determinants of initiation and maintenance of physical activity among older adults: a literature review. Health Psychology Review 2009; 3:147-207

20. den Ouden ME, Schuurmans MJ, Arts IE, van der Schouw YT. Association between physical performance characteristics and independence in activities of daily living in middle-aged and elderly men. Geriatrics \& Gerontology International 2013;13: 274-80

21. Marques EA, Baptista F, Santos DA, Silva AM, Mota J, Sardinha LB. Risk for losing physical independence in older adults: The role of sedentary time, light, and moderate to vigorous physical activity. Maturitas 2014;79: 91-5. doi: 10.1016/j.maturitas.2014.06.012

22. Nejati V, Kordi R, Shoaee F. Evaluation of Effective Motivators and Barriers of Physical Activity in the Elderly. Iranian Journal of Ageing 2010;4: 52-8 [Persian]

23. Nejati V, Ashyeri H. Health related quality of life in the elderly in Kashan. Iranian Journal of Psychiatry and Clinical Psychology 2008;14: 56-61 [Persian]

24. Roh HW, Hong CH, Lee Y, et al. Participation in physical, social, and religious activity and risk of depression in the elderly: a community-based threeyear longitudinal study in Korea. PLOS One 2015;10: e0132838

25. World Health Organization. Physical activity and older adults: recommended levels of physical activity for adults aged 65 and above. 2016. http://www.who.int/dietphysicalactivity/ factsheet_olderadults/en/. Accessed October 7, 2016 26. Garcia PA, Dias JM, Dias RC, Santos P, Zampa CC. A study on the relationship between muscle function, functional mobility and level of physical activity in community-dwelling elderly. Revista Brasileira de Fisioterapia 2011;15: 15-22

27. Payahoo L, Khaje-bishak Y, Pourghasem B, Kabiralavi M-b. The survey of the relationship between quality of life of elderly with depression and 
physicalactivity in Tabriz, Iran. Rehabilitation Medicine 2014;2: 39-46

28. Sanaei M ZS, Norouzi R. The effect of Physical activity on quality of life and Life expectancy in older adults. Sport Management Studies 2014; 5: 137-158 [Persian]

29. Colpani V, Oppermann K, Spritzer PM. Association between habitual physical activity and lower cardiovascular risk in premenopausal, premenopausal, and postmenopausal women: a population-based study. Menopause 2013;20: 525-31

30. Giuli C, Papa R, Mocchegiani E, Marcellini F. Predictors of participation in physical activity for community-dwelling elderly Italians. Archives of Gerontology and Geriatrics 2012;54: 50-4

31. Booth ML, Owen N, Bauman A, Clavisi O, Leslie E. Social-cognitive and perceived environment influences associated with physical activity in older Australians. Preventive medicine 2000;3: 15-22

32. Dumith SC, Gigante DP, Domingues MR. Stages of change for physical activity in adults from Southern Brazil: a population-based survey. International Journal of Behavioral Nutrition and Physical Activity 2007; 4:1-10

33. Moschny A, Platen P, Klaaßen-Mielke R, Trampisch U, Hinrichs T. Barriers to physical activity in older adults in Germany: a cross-sectional study. International Journal of Behavioral Nutrition and Physical Activity 2011; 8:1-10

34. Taghipour M, Hosseini SR, Pouraria S. The Relationship between Physical Activity and Balance Control in the Elderly. Iranian Journal of Ageing 2016;10: 60-7 [Persian]

35. Motefaker $M$, Sadrbafghi $S$, Rafiee $M$, Bahadorzadeh L, Namayandeh S, Karimi M, et al. SuicEpidemiology of physical activity: a population based study in Yazd cityide attempt and its relation to stressors and supportive systems: a study in Karaj city. Tehran University Medical Journal TUMS Publications 2007;65: 77-81 [Persian]

36. Marques EA, Baptista F, Santos DA, Silva AM, Mota J, Sardinha LB. Risk for losing physical independence in older adults: The role of sedentary time, light, and moderate to vigorous physical activity. Maturitas 2014;79: 91-5

37.

http://www.who.int/mediacentre/news/releases/2014/1 ancet-ageing-series/en/

38. Andrieieva O, Hakman A, Kashuba V,Vasylenko M,Patsaliuk K, Koshura A, Istyniuk I. Effects of
Physical Activity on Aging Processes in Elderly Persons. Journal of Physical Education and Sport (JPES) 2019; 19:1308 -14. DOI:10.7752/jpes.2019. s4190

39. Stewart AL, Mills KM, King AC, Haskell WL, Gillis D, Ritter PL. CHAMPS physical activity questionnaire for older adults: outcomes for interventions. Medicine \& Science in Sports \& Exercise 2001;33: 1126-41

40. Sahaf R, Rassafiani M, Fadayevatan R, Delbari A, Saboor M, Mirzaee S, Shams A. Validity and Reliability of CHAMPS Physical Activity Questionnaire for Older People Living in Tehran. SALMAND.2014; 9: 206-217 [Persian]

41. Sadrollahi A, Hosseinian M, Alavi NM, Khalili Z, Esalatmanesh S. Physical activity patterns in the elderly kashan population. Iranian Red Crescent Medical Journal 2016; 18: 1-8

42. Ethisan P, Somrongthong R, Ahmed J, Kumar R Chapman RS. Factors related to physical activity among the elderly population in rural thailand. Journal Prim Care Community Health 2017; 8: 71-6

43. Shiraly R, Shayan Z, Keshtkar V, Hamed M. Selfreported factors associated with engagement in moderate to vigorous physical activity among elderly people: a population-based study. International Journal of Preventive Medicine 2017; 8: 1-16. doi: 10.4103/ijpvm.IJPVM_340_16

44. Momenan A, Delshad M, Mirmiran P, Ghanbarian A, Safarkhani M, Azizi F. Physical inactivity and related factors in an adult Tehranian population (Tehran lipid and glucose study). Iranian Journal of Endocrinology and Metabolism 2012; 13: 493-503 [Persian]

45. Borhaninejad VR, Rashedi V, Tabe R, Delbari A, Ghasemzadeh H. Relationship between fear of falling and physical activity in older adults. Medical Journal of Mashhad University of Medical Sciences 2015; 58: 446-452

46. Eshaghi SR, Shahsanai A, Ardakani MM. Assessment of the Physical Activity of Elderly Population of Isfahan, Iran. Journal of Isfahan Medical School 2011;29: 939-946 [Persian]

47. Milanović Z, Pantelić S, Trajković N, Sporiš G, Kostić R, James N. Age-related decrease in physical activity and functional fitness among elderly men and women. Clinical Interventions in Aging 2013; 8: 549556. doi: 10.2147/CIA.S44112 
48. Ahmadi B, Amini Sanii N, Bani F, Bakhtari F Predictors of Physical Activity in Older Adults in Northwest of Iran. Elderly Health Journal 2018; 4: 75-80 49. Myers AM, Weigel C, Holliday PJ. Sex- and agelinked determinants of physical activity in adulthood. Canadian Journal of Public Health 1989; 80: 256- 60

50. Firebaugh G. Gender differences in exercise and sports. Sociology and Social Research 1989;73: 59-66

51. Kohandel M, Sanatkaran A, Alikaram A. The Comparison of Cognition Performance and Sleep Quality in Older Adults with Different Physical Activity Levels. Strategic studies of sports and youth 2015; 14 [Persian]
52. Sajedi R, Najafian F, Shamsipour Dehkordi P. Interactive effect of physical activity level and gender on inhibitory and behavioral activation systems in the elderly. Journal of Geriatric Nursing. 2019; 5 [Persian] 53. Yarmohammadi S, Mozafar Saadati H, Ghaffari M, Ramezankhani A. A systematic review of barriers and motivators to physical activity in elderly adults in Iran and worldwide. Epidemiol Health. 2019; 41: e2019049 